\title{
Evaluation of Bone Mineral Density, Serum Osteocalcin, and Osteopontin Levels in Postmenopausal Women with Type 2 Diabetes Mellitus, with/without Osteoporosis
}

\author{
Ali B. Roomi $\mathbb{D}^{1,2}$ Abdul-Hassan Mahdi Salih ${ }^{10},{ }^{3}$ Sarmad D. Noori ${ }^{1},{ }^{4}$ Wassan Nori ${ }^{1},{ }^{5}$ \\ and Saba Tariq ${ }^{6}$ \\ ${ }^{1}$ Unviersity of Thi-Qar, Nasiriyah, Thi-Qar 64001, Iraq \\ ${ }^{2}$ Biochemistry and Biological Engineering Research Group, Scientific Research Center, Al-Ayen University, Nasiriyah, \\ Thi-Qar 64001, Iraq \\ ${ }^{3}$ Department of Physiology, College of Medicine, Unviersity of Thi-Qar, Nasiriyah, Iraq \\ ${ }^{4}$ Department of Pharmaceutical Chemistry, College of Pharmacy, Al-Ayen University, Nasiriyah, Thi-Qar, Iraq \\ ${ }^{5}$ Department of Obstetrics and Gynecology, College of Medicine, Mustansiriyah University, Baghdad, Iraq \\ ${ }^{6}$ Department of Pharmacology, University Medical \& Dental College, The University of Faisalabad, Faisalabad 38000, Pakistan
}

Correspondence should be addressed to Ali B. Roomi; dr.ali_bader@alayen.edu.iq

Received 4 December 2021; Revised 16 January 2022; Accepted 29 January 2022; Published 14 February 2022

Academic Editor: Deepak Khajuria

Copyright (c) 2022 Ali B. Roomi et al. This is an open access article distributed under the Creative Commons Attribution License, which permits unrestricted use, distribution, and reproduction in any medium, provided the original work is properly cited.

Objective. Osteoporosis (OP) is a worldwide ailment; we aim to establish new biomarkers in diagnosis by determining the levels of serum osteocalcin and osteopontin along with bone mineral density (BMD) and lumbar T-score, in postmenopausal women with type 2 diabetes mellitus (T2DM) with or without OP. Methods. This observational study included 160 postmenopausal women who were an attendee at outpatient clinics in Al-Hussein Hospital, Thi-Qar province; subdivided into 3 groups based on their T-score testing: Group I $(n=40)$ comprised postmenopausal women without T2DM as controls, Group II $(n=60)$ comprised postmenopausal women with T2DM but without OP, and Group III $(n=60)$ comprised postmenopausal women with T2DM with OP. The dual-energy X-ray absorptiometry was used to measure the BMD (total body, lumbar spine, and femoral) and T-score for lumbar spine and femoral. Glycosylated hemoglobin (HbA1c), fasting blood glucose (FBG), osteocalcin, and osteopontin levels were assessed in all three groups. Results. Compared with controls, Group III demonstrated significantly lower BMD (total body, lumbar spine, and femoral), T-score for lumbar spine and femoral, serum osteocalcin, and osteopontin levels than Group II and Group I $(P<0.001)$. FBG and HbA1c levels were significantly higher in Group III than in Groups I and II $(P<0.001)$. A negative correlation was proved between HbAlc levels with BMD, osteocalcin levels, and osteopontin levels in the three groups. Conclusions. Iraqi postmenopausal women with T2DM had a significantly lower bone mineral density, serum osteocalcin, and osteopontin levels. These results may serve as adjuvants in screening for OP, particularly among diabetic patients.

\section{Introduction}

Osteoporosis (OP) is a common bone illness that affects people worldwide and is characterized by reduced bone mineral density (BMD) and deteriorated bone tissues, making the bones fragile $[1,2]$. Diabetes mellitus (DM) is a metabolic condition characterized by elevated blood glucose levels due to insulin resistance, deficiency, or both [3]. Type 2 DM (T2DM), characterized by hyperglycemia, accounts for about $90 \%$ of all diabetes cases worldwide [4]. Patients with type 1 DM have a lower BMD whereas those with T2DM are at a higher risk of experiencing a bone fracture, given their high or average $\mathrm{BMD}$ as measured by dualenergy X-ray absorptiometry (DXA) $[4,5]$. Other causes that contribute to the development of fractures in patients with T2DM include sarcopenia, impaired balance due to peripheral neuropathy, diabetic retinopathy, and medications used in diabetes $[6,7]$. 
The postmenopausal period is often related to OP because of the hypoestrogenic condition induced by ovarian failure [3, 8]. OP is better prevented than treated; the enhancement of public understanding of its consequences and risks by the media and community newsletter agencies has contributed to a fair reduction in many countries that have embraced this approach [9-11].

Osteocalcin (OC) is a small bone-specific noncollagen protein found chiefly in the bone and is produced by osteoblasts. OC is a sensitive marker of bone formation. OC is carboxylated and mostly combined within the bone extracellular matrix; however, a small proportion is released into the circulation where OC plays a role in fat and glucose metabolism [12]. Osteopontin (OPN) is a noncollagenous matrix protein in bone tissues and is involved in bone remodeling and stimulates bone resorption $[11,13]$. The association of OP biomarkers with T2DM is well known, but studies from Thi-Qar, Iraq, are few. This is a leading study in south Iraq, Thi-Qar governorate, that aims to evaluate the BMD and serum OC and OPN levels in postmenopausal women with T2DM along with selected demographic criteria.

\section{Materials and Methods}

In this observational study, we included 210 postmenopausal women who attended Al-Hussein Teaching Hospital and private clinics in Thi-Qar Province, Iraq. After the exclusions, 160 postmenopausal women remained. The volunteers were sampled between November 2019 and March 2020. All of them underwent a physical exam, blood sampling, and bone mineral density measurement. Volunteers in this study were postmenopausal women based on the World Health Organization definition for menopause including those who had their last menstrual period at least one year ago [14]. Females with rheumatic disease, malignancy, gout, hypertension, history of smoking, history of alcohol consumption, and abnormal renal, liver, or thyroid function were excluded. Similarly, females receiving medications that impact bone metabolism (bisphosphonates, strontium translate, selective estrogen receptor modulators, calcitonin, cathepsin-K inhibitors, and parathyroid hormone) were also excluded. DXA scan was used to measure the T-score value at the lumbar spine (LS: L1-L4) and femoral neck (FN). All scans were conducted using the DXA scanner by DEXXUM3 (Osteosys Co. Ltd., Korea) at Al-Hussein Teaching Hospital. T-score value of 0 to 1 was regarded as normal BMD, -1 to -2.5 was considered as osteopenia, and $\leq-2.5$ was estimated as OP [15]. The participants in this study were divided into 3 groups: Group I, 40 postmenopausal women as controls; Group II, 60 postmenopausal women with T2DM but without OP; and Group III, 60 postmenopausal women with T2DM with OP based on their T-score.

A five $\mathrm{mL}$ fasting blood sample was collected from each participant and divided into 2. A two $\mathrm{mL}$ portion was placed in an EDTA tube and was used to measure glycated hemoglobin (HbAlc), and the other three $\mathrm{mL}$ was allowed to clot and then centrifuged at $3600 \mathrm{rpm}$ for $10 \mathrm{~min}$. The serum was separated from the coagulants and stored at $-20^{\circ} \mathrm{C}$.
Serum OC and OPN levels were estimated using an enzymelinked immunosorbent assay (ELISA) kit provided by Demeditec Diagnostics GmbH, Germany. Serum fasting blood glucose (FBG) concentration was estimated according to the Copper assay kit (Randox, England) by using the spectrophotometer. The precipitate was evaluated by measuring the red (total hemoglobin) and the blue (glycosylated hemoglobin) color intensity with the NycoCard Reader (Axis-Shield Co., Norway).

\section{Statistical Analysis}

All statistical analyses were performed by using the program IBM (SPSS) version 26. The data in this study were shown as mean \pm SD. One-way ANOVA test was used to compare the three study groups. Significance was set for a $P$ value of $<0.01$. Pearson's correlation coefficient tested the correlation between $\mathrm{HbA} 1 \mathrm{c}$ as a dependent variable and $\mathrm{BMD}$, $\mathrm{OC}$, and OPN in three groups. The correlation coefficient is positive if the 2 variables under consideration tend to increase or decrease together, and the larger the value, the closer the association. Conversely, the correlation coefficient is negative if an increasing value of 1 variable tends to go with a decreasing value for the other and vice versa.

\section{Results}

The general characteristics of postmenopausal women are shown in Table 1. Markers such as BMD LS $\left(0.57 \pm 0.10 \mathrm{~g} / \mathrm{cm}^{2}\right)$, T-score $(-3.60 \pm 1.80)$, serum OC $(19.30 \pm 1.72 \mathrm{ng} / \mathrm{mL})$, and OPN $(14.2 \pm 1.40 \mathrm{ng} / \mathrm{mL})$ were significantly lower in Group III compared to BMD $(0.78 \pm 0.10)$, T-score $(-2.01 \pm 0.90)$, serum OC $(24.40 \pm 2.32)$, and OPN $(18.10 \pm 2.01)$ in Group II and BMD $(0.98 \pm 0.12), \quad$ T-score $(0.92 \pm 0.12)$, serum OC $924.40 \pm 2.61$, and OPN $(19.40 \pm 2.60)$ in Group I, with $P<0.001$.

In Group III, FBG $(8 \pm 1.4 \mathrm{mmol} / \mathrm{L})$ and $\mathrm{HbA} 1 \mathrm{c}$ $(8.01 \pm 2.01 \%)$ were significantly higher compared to those in Group II $(5.01 \pm 1.20$ and $7.12 \pm 1.60)$ and Group I $(4.10 \pm 1.01$ and $5.01 \pm 1.41)$, respectively. A significantly lower BMD LS, BMD FN, and total body BMD were observed in Group III women compared with those in Groups I and II. Serum FBG and HbA1c were highest in Group III. FBG was significantly lower in Group II compared with Group I. HbA1c level, taken as a dependent marker, was calculated by linear regression analysis against T-score, OC, and OPN as independent markers. The results of $\mathrm{HbAlc}$ showed a significant negative correlation with BMD, OC, and OPN in three groups as illustrated in Table 2.

\section{Discussion}

T2DM is a chronic metabolic disease that has been associated with various risk factors. Diabetes and OP frequently coexist in elderly individuals; this unhealthy alliance affects the quality of life. The incidence of osteoporosis continues to rise along with fragility fractures [16]. The objective of the study was to see the relationship between BMD, OC, and 
TABLE 1: Characteristics of postmenopausal women according to groups.

\begin{tabular}{|c|c|c|c|c|}
\hline Parameter mean $\pm \mathrm{SD}$ & Group I $(n=40)$ & Group II $(n=60)$ & Group III $(n=60)$ & $P$ value \\
\hline Age (years) & $58.20 \pm 6.01$ & $58.70 \pm 5.01$ & $57.91 \pm 5.40$ & 0.22 \\
\hline Menopause duration (years) & $9.21 \pm 4.61$ & $8.92 \pm 4.22$ & $9.01 \pm 4.40$ & 0.14 \\
\hline T2DM duration (years) & - & $7.21 \pm 5.3$ & $7.81 \pm 5.60$ & 0.12 \\
\hline BMI $\left(\mathrm{kg} / \mathrm{m}^{2}\right)$ & $24.90 \pm 1.01$ & $26.10 \pm 2.61$ & $27.01 \pm 2.40$ & 0.19 \\
\hline Total body BMD $\left(\mathrm{g} / \mathrm{cm}^{2}\right)$ & $0.91 \pm 0.14$ & $0.80 \pm 0.10$ & $0.60 \pm 0.12$ & $<0.001^{*}$ \\
\hline BMD LS $\left(\mathrm{g} / \mathrm{cm}^{2}\right)$ & $0.98 \pm 0.12$ & $0.78 \pm 0.10$ & $0.57 \pm 0.10$ & $<0.001^{*}$ \\
\hline BMD FN $\left(\mathrm{g} / \mathrm{cm}^{2}\right)$ & $0.61 \pm 0.12$ & $0.52 \pm 0.14$ & $0.43 \pm 0.17$ & $<0.001^{*}$ \\
\hline T-score LS & $0.92 \pm 0.12$ & $-2.01 \pm 0.90$ & $-3.60 \pm 1.80$ & $<0.001^{*}$ \\
\hline T-score FN & $0.83 \pm 0.16$ & $-1.98 \pm 0.13$ & $-3.94 \pm 1.15$ & $<0.001^{*}$ \\
\hline Fasting glucose (mmol/L) & $4.10 \pm 1.01$ & $5.01 \pm 1.20$ & $8.01 \pm 1.41$ & $<0.001^{*}$ \\
\hline HbAlc (\%) & $5.01 \pm 1.41$ & $7.12 \pm 1.60$ & $8.01 \pm 2.01$ & $<0.001^{*}$ \\
\hline $\mathrm{OC}(\mathrm{ng} / \mathrm{mL})$ & $24.40 \pm 2.61$ & $24.40 \pm 2.32$ & $19.30 \pm 1.72$ & $<0.001^{*}$ \\
\hline OPN (ng/mL) & $19.40 \pm 2.60$ & $18.10 \pm 2.01$ & $14.2 \pm 1.40$ & $<0.001^{*}$ \\
\hline
\end{tabular}

BMI, body mass index; BMD, bone mineral density; LS, lumbar spine; FN, femoral neck; HbA1c, hemoglobin A1c; OC, osteocalcin; OPN, osteopontin. *indicates significance.

TABLE 2: The correlations of HbA1c level versus BMD, OC, and OPN in the studied subgroups.

\begin{tabular}{lccccccc}
\hline Studied & \multicolumn{2}{c}{ BMD } & \multicolumn{2}{c}{ OC } & \multicolumn{2}{c}{ OPN } \\
groups & $r$ & $P$ value & $r$ & $P$ value & $r$ & $P$ value \\
\hline Group I & -0.312 & $<0.001^{*}$ & -0.381 & $<0.001^{*}$ & -0.341 & $<0.001^{*}$ \\
Group II & -0.345 & $<0.001^{*}$ & -0.391 & $<0.001^{*}$ & -0.347 & $<0.001^{*}$ \\
Group III & -0.478 & $<0.001^{*}$ & -0.454 & $<0.001^{*}$ & -0.431 & $<0.001^{*}$
\end{tabular}

${ }^{*}$ Correlation is at significant level at 0.001 .

OPN levels in postmenopausal females with type $2 \mathrm{DM}$ with and without osteoporosis.

We found that BMD and serum levels of OC and OPN were significantly lower in postmenopausal females with type 2 DM with OP. Similar to our results, another study found a decreased level of OC in the postmenopausal osteoporotic group [17]. In contrast to our results, another study found a significant negative association of serum OC with BMD [18]. However, the major difference was that our patients were diabetic and there could be possible interaction of OC with glucose metabolism showing some energy metabolism disorders in our females.

We also could not find any association of BMI with OP. Similar to our results, few other studies could not establish any association of BMI with OP $[19,20]$. In contrast, evidence also suggests that increased BMI has a protective role against $\mathrm{OP}$ as an increase in mechanical loading of bones leads to increased bone mass [21].

T-score LS and T-score FN in Group III were significantly lower especially in postmenopausal women with T2DM, in line with few other studies which had shown significantly reduced BMD is in patients suffering from T2DM [22]. In this study, the mean $\mathrm{HbAlc}$ and serum FBG were significantly increased in patients with OP. Our results support the idea that chronic hyperglycemia reduces bone quality by inhibiting OC secretion and increasing bone resorption. Another point to consider is diabetes-related comorbidities as diabetic retinopathy and peripheral neuropathy are responsible for increased falls and thus increased fractures. There is conflicting evidence that there is a relationship between insulin or other oral medication and raised risk of osteoporosis [6-9]. Moreover, antidiabetic therapy was blamed to increase OP risk since insulin increases fall by hypoglycemic attacks $[6,22]$. The use of thiazolidinediones has been associated with increased fracture risks in both men and women with T2DM as it inversely affects the bone quality by suppressing the differentiation of mesenchymal stem cells to osteoblasts in favor of differentiation to adipocytes $[6,23]$.

$\mathrm{OC}$ is a bone-derived hormone which is involved in bone formation and calcium homeostasis. It protects against high-fatinduced obesity and insulin resistance. It had been observed that patients with OP showed a substantial decrease in serum OC, and these levels were positively related to poor regulated DM and are inversely related to FBG. Moreover, lower serum OC was associated with increased mortality in women with T2DM [22-24]. This study confirmed an inverse correlation between $\mathrm{HbAlc}$ and $\mathrm{OC}$ levels in line with another study who reported a significant inverse relation between serum OC level and forearm BMD in patients with knee osteoarthritis (KOA) [25].

Impaired bone formation and uncoupling of bone turnover was evident even in postmenopausal patients with rheumatoid arthritis and increased the risk of hip fracture [26]. There was a significant decrease in the T-score of the hip and serum levels of OC in postmenopausal Egyptian women with obesity [27]. The last conclusions were consistent with our findings, where an inverse correlation was proven between BMD and OC levels.

OPN, a multifunctional protein primarily associated with bone metabolism, bone remodeling, and bone turnover proved to be inversely related to $\mathrm{HbAlc}$. OPN levels tend to rise as glucose tolerance deteriorates in humans [13]. Earlier studies support our findings assuming a positive correlation with HbA1c, FBG, 2-hour plasma glucose, and OPN [13]. Furthermore, OPN was found to be related to progressive joint damage in KOA and serves as a biochemical marker for determining disease severity [28].

In Iraqi postmenopausal females, the incidence of $\mathrm{OP}$ increased to $22.8 \%$ [29]. This study highlights the need to establish risk prediction models to avoid consistently underestimating the risk of OP-related fractures in patients with diabetes. There is an emerging need for fracture prevention approaches in patients with T2DM. Several Arab countries have acknowledged the problem and have considered taking 
initiatives to minimize its dramatic impact on the wellbeing of women and health costs. The introduction of several effective pharmaceutical agents to treat those at high risk has yielded a promising basis for conducting public health education [9]. The decline in hip fracture in Lebanon could be because of multiple factors: updating the first Lebanese OP assessment and therapeutic guidelines in 2007 along with the pervasive use of calcium and vitamin D supplements. OP rates in Morocco and Kuwait remain stable, slowly rising from 2009 to 2012, and can be ascribed to an increase in the population group aged $\geq 50$ years [10]. The United States recently noted a higher incidence of OP; a decline in DXA screening could be a major factor, along with a decline in bisphosphonate drug prescription. The US Food and Drug Administration updated amended labeling for risk of OP and atypical femur fractures [30-32].

Limitations of this study include the small sample size used as the hospital was transformed into a coronavirus disease 2019 emergency hospital during the pandemic. Moreover, the socioeconomic status of the participants should have been taken into account so that more access to earlier diagnostic and preventive strategies would have presented those with a degree of osteopenia rather than those with OP. The novelty of this work lies in the fact that it tackled the issue of OP despite the lack of public knowledge of its serious complications, the limited funding, the poor socioeconomic status of many Thi-Qar residents, and the high cost of DXA scanning along with the lack of vitamin and mineral supplements.

\section{Conclusions}

This study has proven that Iraqi postmenopausal women in Thi-Qar Province had a reduced bone mineral density along with a significant reduction in serum OPN and OC in postmenopausal women with diabetes. These parameters may be used as adjuvants in screening for the risk of $\mathrm{OP}$ with particular emphasis on patients with diabetes to ensure that elderly Iraqi women grow crowned with health.

\section{Data Availability}

The data will be provided upon reliable reason from the corresponding author.

\section{Ethical Approval}

The study was approved by the Al-Hussein Teaching Hospital's Institutional Research Ethics Committee, Thi-Qar province. Written informed consent was obtained from all participants.

\section{Conflicts of Interest}

The authors declare no conflicts of interest to any party.

\section{Acknowledgments}

The authors wish to thank the staff of Al-Hussein Teaching Hospital who supported the collection of samples.

\section{References}

[1] O. Kaewboonchoo, F. C. Sung, C. L. Lin, H. C. Hsu, and C. T. Kuo, "Risk of osteoporosis and fracture in victims with burn injury," Osteoporosis International, vol. 30, no. 4, pp. 837-843, 2019.

[2] S. C. DeShields and T. D. Cunningham, "Comparison of osteoporosis in US adults with type 1 and type 2 diabetes mellitus," Journal of Endocrinological Investigation, vol. 41, no. 9, pp. 1051-1060, 2018.

[3] S. H. Al-Badry, S. A. Khalaf, and A. L. Al-Khuzaiy, "The effects of irisin on the transforming growth factor-betal (TGF- $\beta 1$ ) level in type-1 diabetic patients in Thi-Qar province," International Journal of Pharmaceutical Research, vol. 12, no. 4, pp. 2523-2529, 2020.

[4] O. N. Alhuzaim, E. J. H. Lewis, L. E. Lovblom et al., "Bone mineral density in patients with longstanding type 1 diabetes: results from the Canadian Study of Longevity in Type 1 Diabetes," Journal of Diabetes and Its Complications, vol. 33, no. 11, Article ID 107324, 2019.

[5] E. A. Hafez, S. A. ElBakry, S. I. Ibrahim, C. S. Morad, S. A. Hamza, and D. M. Abd El-Khalik, "Assessment of fracture risk in a cohort of Egyptian female Systemic Lupus erythematosus patients," The Egyptian Rheumatologist, vol. 40, no. 2, pp. 85-91, 2018.

[6] A. B. Roomi, R. M. H. AL-Salih, and S. A. Ali, "Impact metformin and insulin therapy on parathyroid hormone and $25(\mathrm{OH})$ vitamin $\mathrm{D}$ in diabetic post-menopausal Iraqi women," Journal of Physics: Conference Series, vol. 1279, no. 1, Article ID 012008, 2019.

[7] D. S. H. Bell and E. Goncalves, "Why do falls and lower limb fractures occur more frequently in the diabetic patient and how can they be prevented?" Diabetes Therapy, vol. 11, no. 8, pp. 1687-1694, 2020.

[8] A. Badr Roomi, W. Nori, and R. Mokram Hamed, "Lower serum irisin levels are associated with increased osteoporosis and oxidative stress in postmenopausal," Reports of Biochemistry and Molecular Biology, vol. 10, no. 1, pp. 13-19, 2021.

[9] A. B. Roomi, W. Nori, and S. H. Al-Badry, "The value of serum adiponectin in osteoporotic women: does weight have an effect?" Journal of Obesity, vol. 2021, Article ID 5325813, 5 pages, 2021.

[10] R. K. Saad, H. Harb, I. R. Bou-Orm, W. Ammar, and G. El-Hajj Fuleihan, "Secular trends of hip fractures in Lebanon, 2006 to 2017: implications for clinical practice and public health policy in the Middle East region," Journal of Bone and Mineral Research, vol. 35, no. 1, pp. 71-80, 2020.

[11] S. S. El-Tawab, E. K. A. Saba, H. M. T. Elweshahi, and M. H. Ashry, "Knowledge of osteoporosis among women in Alexandria (Egypt): a community based survey," The Egyptian Rheumatologist, vol. 38, no. 3, pp. 225-231, 2016.

[12] S. B. Oh, W. Y. Lee, H.-K. Nam, Y.-J. Rhie, and K.-H. Lee, "Serum osteocalcin levels in overweight children," Annals of Pediatric Endocrinology \& Metabolism, vol. 24, no. 2, pp. 104-107, 2019.

[13] C. De Fusco, A. Messina, and V. Monda, "Osteopontin: relation between adipose tissue and bone homeostasis," Stem Cells International, vol. 2017, Article ID 4045238, 6 pages, 2017.

[14] E. B. Gold, "The timing of the age at which natural menopause occurs," Obstetrics \& Gynecology Clinics of North America, vol. 38, no. 3, pp. 425-440, 2011. 
[15] J. A. Kanis, Assessment of Osteoporosis at the Primary HealthCare Level, WHO Collaborating Centre for Metabolic Bone Diseases, Sheffield, UK, 2007.

[16] M. Catoire and S. Kersten, "The search for exercise factors in humans," The FASEB Journal, vol. 29, no. 5, pp. 1615-1628, 2015.

[17] N. M. Al-Daghri, S. Yakout, and E. Al-Shehri, "Inflammatory and bone turnover markers in relation to PTH and vitamin D status among Saudi postmenopausal women with and without osteoporosis," International Journal of Clinical and Experimental Medicine, vol. 7, no. 9, pp. 2812-2819, 2014.

[18] S. Singh, D. Kumar, and A. K. Lal, "Serum osteocalcin as a diagnostic biomarker for primary osteoporosis in women," Journal of Clinical and Diagnostic Research : Journal of Clinical and Diagnostic Research, vol. 9, no. 8, pp. RC04-7, 2015.

[19] S. Tariq, S. Tariq, and S. S. Alam, "Effect of Ibandronate treatment on homocystein and leptin level in postmenopausal osteoporotic females," Osteoporosis International, vol. 26, pp. 1187-1192, 2015.

[20] S. Tariq, S. Tariq, K. P. Lone, and S Khaliq, "Alkaline phosphatase is a predictor of Bone Mineral Density in postmenopausal females," Pakistan journal of medical sciences, vol. 35, no. 3, pp. 749-753, 2019.

[21] S. Tariq, S. Tariq, and K. P. Lone, "Relationship of anthropometric measures with bone mineral density in postmenopausal non-osteoporotic, osteopenic and osteoporotic women," The Journal of the Pakistan Medical Association, vol. 67, no. 4, pp. 590-594, 2017.

[22] I. Kanazawa, M. Notsu, H. Miyake, K. Tanaka, and T. Sugimoto, "Assessment using serum insulin-like growth factor-I and bone mineral density is useful for detecting prevalent vertebral fractures in patients with type 2 diabetes mellitus," Osteoporosis International, vol. 29, no. 11, pp. 2527-2535, 2018.

[23] A. Moayeri, M. Mohamadpour, S. Mousavi, E. Shirzadpour, S. Mohamadpour, and M. Amraei, "Fracture risk in patients with type 2 diabetes mellitus and possible risk factors: a systematic review and meta-analysis," Therapeutics and Clinical Risk Management, vol. Volume 13, pp. 455-468, 2017.

[24] S. A. Paschou, A. D. Dede, P. G. Anagnostis, A. Vryonidou, D. Morganstein, and D. G. Goulis, "Type 2 diabetes and osteoporosis: a guide to optimal management," Journal of Clinical Endocrinology \& Metabolism, vol. 102, no. 10, pp. 3621-3634, 2017.

[25] W. A. A. Elwakil, D. Mohasseb, D. Elkaffash, S. Elshereef, and M. Elshafey, "Serum leptin and osteoporosis in postmenopausal women with primary knee osteoarthritis," The Egyptian Rheumatologist, vol. 38, no. 3, pp. 209-215, 2016.

[26] T. Gheita, S. Fawzy, A. Rizk, and H. Hussein, "Impaired bone formation and osteoporosis in postmenopausal elderly onset rheumatoid arthritis patients," The Egyptian Rheumatologist, vol. 33, no. 3, pp. 155-162, 2011.

[27] S. E. Ibrahim, H. F. ElShishtawy, A. Helmy, Z. A. Galal, and M. H. Abdel Salam, "Serum leptin concentration, bone mineral density and bone biochemical markers in a sample of Egyptian women: a possible relationship," The Egyptian Rheumatologist, vol. 33, no. 4, pp. 171-177, 2011.

[28] F. I. Mohammed, M. I. Abd El-Azeem, and A. M. KamalElDin, "Plasma and synovial fluid osteopontin levels in patients with knee osteoarthritis: relation to radiological grade," The Egyptian Rheumatologist, vol. 34, no. 3, pp. 131-136, 2012.

[29] T. A. Gheita and N. Hammam, "Epidemiology and awareness of osteoporosis: a viewpoint from the Middle East and North
Africa," International Journal of Clinical Rheumatology, vol. 13, no. 3, pp. 134-147, 2018.

[30] H. Miyake, I. Kanazawa, and T. Sugimoto, "Association of bone mineral density, bone turnover markers, and vertebral fractures with all-cause mortality in type 2 diabetes mellitus," Calcified Tissue International, vol. 102, no. 1, pp. 1-13, 2018.

[31] C. Klop, D. Gibson-Smith, P. J. M. Elders et al., "Anti-osteoporosis drug prescribing after hip fracture in the UK: 2000-2010," Osteoporosis International, vol. 26, no. 7, pp. 1919-1928, 2015.

[32] S. C. Kim, D. H. Kim, H. Mogun et al., "Impact of the U.S. Food and drug administration's safety-related announcements on the use of bisphosphonates after hip fracture," Journal of Bone and Mineral Research, vol. 31, no. 8, pp. 1536-1540, 2016. 